\title{
Tratamiento antiinflamatorio en el síndrome de Stevens-Johnson y la necrólisis epidérmica tóxica: revisión sistemática de la literatura científica
}

Anti-inflammatory treatment in adults with Stevens Johnson Syndrome and toxic epidermal necrolysis: a systematic review

\author{
Diana Carrillo, Luz Zárate', Ana Ramírez², Carlos Serrano ${ }^{1,3}$ \\ 1. Departamento de Medicina Interna, Fundación Valle del Lili, Cali, Colombia \\ 2. Departamento de Dermatología, Fundación Valle del Lili, Cali, Colombia \\ 3. Unidad de Alergia, Fundación Valle del Lili, Cali, Colombia
}

\section{Resumen}

Antecedentes. No existe un tratamiento específico para el manejo de la necrólisis epidérmica tóxica y el síndrome de Stevens-Johnson. Los pacientes son tratados, principalmente, mediante la eliminación del factor desencadenante y medidas de soporte. Nuestro objetivo fue hacer una revisión sistemática para evaluar el efecto del tratamiento antiinflamatorio en adultos con síndrome de Stevens-Johnson o necrólisis epidérmica tóxica.

Métodos. Se hizo una búsqueda sistemática, objetiva y reproducible de estudios originales en bases de datos electrónicas como Cochrane Skin Group Specialized Register, Cochrane Controlled Trials and CDSR, Trip database, MEDLINE, EMBASE, CINAHL, Biomed, NCBI, HINARI y LILACS. Se usaron los términos clave "Stevens-Johnson syndrome / drug therapy" o "Stevens-Johnson syndrome / therapy" o "Toxic epidermal necrolysis / drug therapy" o "Toxic epidermal necrolysis / therapy", con restricción a ensayos abiertos y estudios clínicos de fase III desde 1998 hasta el 30 de diciembre de 2011, y a idioma español e inglés. No se incluyó literatura gris y se revisaron títulos y resúmenes para identificar los ensayos clínicos.

Resultados. Se encontró un solo un ensayo clínico controlado con uso de talidomida y siete ensayos abiertos sin grupo control o con un control histórico. Los fármacos usados fueron inmuglobulina en cuatro estudios, ciclosporina en dos y metilprednisolona en uno. A pesar del extenso compromiso de la piel, de más del $50 \%$, la mortalidad fue de o $\%$ en los dos estudios con ciclosporina, y hubo una muerte en el grupo con metilprednisolona y una en el grupo de inmunoglobulina. La mortalidad en el grupo con talidomida fue de $83 \%$. En un estudio con dosis altas de inmunoglobulina, la mortalidad observada fue de $32 \%$ Vs. $21 \%$ de la mortalidad predicha y, en el estudio con bajas dosis de inmunoglobulina, la mortalidad predicha fue de $32 \%$ comparada con la mortalidad observada del $10 \%$.

Conclusión. Parece existir algún beneficio en el tratamiento con metilprednisolona, inmunoglobulina y ciclosporina, pero se requieren ensayos clínicos controlados para determinar la eficacia de estos fármacos. La talidomida aumentó la mortalidad en los pacientes con necrólisis epidérmica tóxica.

PALABRAS CLAVE: síndrome de Stevens-Johnson, necrólisis epidérmica toxica, tratamiento.

\section{Correspondencia:}

Carlos Serrano

Email:

cd_serrano@yahoo.com

Recibido: 2 de septiembre de 2012.

Aceptado: 8 de noviembre de 2012.

No se reportan conflictos de intereses. 


\section{Summary}

Background: There is no specific treatment approved for toxic epidermal necrolysis and Stevens Johnson syndrome. Patients must be treated mainly by eliminating the triggering factor and general support. Our aim was to perform a systematic review to evaluate the effect of anti-inflammatory treatment in adults with Stevens Johnson syndrome or toxic epidermal necrolysis.

Methods: Systematic, objective, exhaustive and reproducible search of original research was conducted in electronic databases (Cochrane Skin Group Specialized Register, the Cochrane Controlled Trials and CDSR, Trip database, MEDLINE, EMBASE, CINAHL, Biomed, NCBI, HINARI and LILACS using "StevensJohnson Syndrome / drug therapy" or "Stevens-Johnson Syndrome / therapy" or "Toxic epidermal necrolysis / drug therapy" or "Toxic epidermal necrolysis / therapy" as key words with restriction to open trials and phase III clinical trials from 1998 to December 30, 2011 in Spanish or English languages. Grey literature was not included. Title or abstract were reviewed to identify clinical trials.

Results: Only one randomized, double-blind, placebo-controlled study with thalidomide was identified, and 7 open clinical trials without a control group or historical control were included. The drug therapy used was immunoglobulin in four studies, cyclosporine in two, and methylprednisolone in one. Although the extensive skin detachment of more than $50 \%$, mortality was $0 \%$ in two studies with cyclosporine, one death in the methylprednisolone group and one death in the immunoglobulin group. Mortality in the thalidomide group was $83 \%$. In one study with high-dose of immunoglobulin, the observed mortality was $32 \%$ vs. $21 \%$ of predicted mortality and in the low-dose of immunoglobulin, predicted mortality was $32 \%$ compared to $10 \%$ of observed mortality.

Conclusion: There seems to be some benefit from therapy with methylprednisolone, immunoglobulin and cyclosporine but randomized controlled clinical trials are needed to determine the efficacy of these therapies available. Thalidomide increased mortality in toxic epidermal necrolysis.

KEYWORDS: Stevens-Johnson syndrome, toxic epidermal necrolysis; drug therapy.

\section{Introducción}

El síndrome de Stevens-Johnson y la necrólisis epidérmica tóxica son reacciones mucocutáneas agudas, graves y potencialmente mortales. Los medicamentos son el factor relacionado en el $80 \%$ de $\operatorname{los} \operatorname{casos}^{1-5} \mathrm{y}$ en una minoría se asocian con enfermedades infec$\operatorname{ciosas}^{3,6-8}$. Se consideran formas graves del espectro de reacciones adversas epidermolíticas ${ }^{9,10}$ y se diferencian según el porcentaje de superficie corporal que comprometen. Esta es de menos del $10 \%$ en el síndrome de Stevens-Johnson, de 10 a $30 \%$ en la superposición síndrome de Stevens-Johnson/necrólisis epidérmica tóxica y de más del $30 \%$ en la necrólisis epidérmica tóxica ${ }^{1,3,10}$. Se pueden presentar a cualquier edad, con una incidencia de 1,2 a 6 casos por millón personas por año para el síndrome de Stevens-Johnson y de 0,4 a 1,2 casos por millón de personas por año ${ }^{2,3}$ para la necrólisis epidérmica tóxica en países industrializados, y es 1.000 veces más frecuente en pacientes con VIH (un caso por cada 1.000 personas por año) ${ }^{1,11,12}$. La mortalidad varía entre 1 y $35 \%$; es de $5 \%$ en el síndrome de Stevens-Johnson ${ }^{5}$ y de 30 a $60 \%$ en la necrólisis epidérmica tóxica ${ }^{1,5,10}$. Batsuji-Garin, et al. ${ }^{13}$, reportaron una escala de predicción de mortalidad (SCORTEN) que incluye la edad, el antecedente de neoplasia maligna, la frecuencia cardiaca, la superficie corporal comprometida, el valor de nitrógeno ureico, la glucemia y el nivel de bicarbonato. La puntuación va de o a 7 y predice una mortalidad de 3,2 \% para aquellos con una puntuación entre o a $1 \mathrm{y}$, de más del $90 \%$ para aquellos con una puntuación mayor de $5^{14}$.

Hasta el momento, no hay un tratamiento específico 
aprobado para estas enfermedades ${ }^{10}$. El manejo se basa en la identificación y eliminación del fármaco desencadenante y en la instauración de medidas de soporte en una unidad de cuidado intensivo o unidad de quemados $^{1-3,10}$. Se han publicado estudios observacionales y experimentales utilizando diferentes modalidades terapéuticas antiinflamatorias, teniendo en cuenta la fisiopatología de estas enfermedades ${ }^{1,2}$. Por todo lo anterior, el objetivo de hacer una revisión sistemática fue determinar la reducción de la mortalidad, la presencia de secuelas y los eventos adversos relacionados con los diferentes tratamientos antiinflamatorios disponibles, en pacientes con síndrome de Stevens-Johnson y necrólisis epidérmica tóxica.

\section{Métodos}

Se hizo una revisión sistemática de la literatura científica que incluyó ensayos clínicos controlados así como estudios abiertos, debido a que en una revisión sistemática previa ${ }^{15}$ solo se encontró un ensayo clínico controlado que evaluaba el efecto de las alternativas terapéuticas antiinflamatorias en el síndrome de Stevens-Johnson y la necrólisis epidérmica tóxica. Se incluyeron adultos mayores de 18 años con diagnóstico de síndrome de Stevens-Johnson, necrólisis epidérmica tóxica o síndromes de superposición de síndrome de Stevens-Johnson/necrólisis epidérmica tóxica.

Se definió el síndrome de Stevens-Johnson como la formación de lesiones eritematosas en piel, púrpura o lesiones en "diana" atípicas que comprometieran menos del $10 \%$ de la superficie corporal, con compromiso de mucosas. Se definió como necrólisis epidérmica tóxica la presencia de lesiones similares a las del síndrome de Stevens-Johnson, pero que comprometieran más de $30 \%$ de la superficie corporal, asociadas a síntomas sistémicos. El síndrome de superposición síndrome de Stevens-Johnson/necrólisis epidérmica tóxica se diagnosticó cuando el compromiso era de 10 a $30 \%$ de la superficie corporal, asociado con síntomas sistémicos.

Se hizo una búsqueda objetiva y reproducible de trabajos originales en bases de datos electrónicas: Cochrane Skin Group Specialized Register, Cochrane Controlled Trial, CDSR, Tripdatabase, MEDLINE, EMBASE, CINAHL, BIOMED, NCBI, HINARI, LILACS y OMIN, utilizando los términos "Stevens-Johnson syndrome/drug therapy" o "Stevens-Johnson syndrome/ therapy" o "Toxic epidermal necrolysis/drug therapy" o "Toxic epidermal necrolysis/therapy", los cuales permitieron identificar estudios de tratamiento en el síndrome de Stevens-Johnson o en la necrólisis epidérmica tóxica, con restricción a ensayos abiertos y ensayos clínicos de fase III, desde 1998 hasta el 30 de diciembre de 2011, e idioma español o inglés. No se incluyó literatura gris.

Dos personas revisaron el título o el resumen de cada artículo, con el fin de establecer cuáles eran ensayos clínicos. Se hizo la exploración detallada del artículo cuando el resumen no especificaba las características del estudio o cuando existieron dudas sobre el tipo de estudio o de las medidas de resultado. Para los artículos que cumplieron las características descritas, se utilizaron los criterios de calidad de acuerdo con la puntuación de Oxford.

De los artículos seleccionados, se obtuvo el puntaje SCORTEN (Score of Toxic Epidermal Necrolysis) o escala de gravedad fisiológica, el tratamiento utilizado, la duración del mismo, la dosis, la frecuencia de uso, la disminución o reducción de síntomas, el tiempo de hospitalización, la mortalidad observada, las complicaciones, las secuelas y los eventos adversos, mediante un formato de recolección elaborado en Microsoft Office Excel $2007^{\circledR}$. En algunos casos, se calculó el puntaje del SCORTEN de acuerdo con lo reportado en el artículo. No se hizo un metaanálisis, teniendo en cuenta que hubo estudios experimentales sin grupo de comparación.

\section{Resultados}

Ocho estudios cumplieron los criterios de inclusión ${ }^{16-24}$. Hubo un artículo al cual no se tuvo acceso ni al resumen ni al contenido y no se pudo determinar si se trataba de un ensayo abierto o de fase III $^{25}$. Asimismo, se excluyó un artículo en el que no se evaluó la mortalidad sino la recuperación oftalmológica ${ }^{24} \mathrm{y}$ otro que era una cohorte retrospectiva ${ }^{16}$ (FIGURA 1).

Solo hubo un ensayo clínico de asignación aleatoria con doble enmascaramiento, controlado con placebo, en el que se evaluó el efecto de la talidomida ${ }^{19}$. Otro fue un ensayo clínico con asignación aleatoria y un solo enmascaramiento para aquellos que revisaron las biopsias de piel tomadas los días o, 5 y 7, en el cual se compararon seis pacientes que recibieron inmunoglobulina (dos), ciclosporina (dos), y tratamiento de soporte $(\mathrm{dos})^{21}$. Los otros seis estudios fueron experimentales, en los cuales se recolectó, de forma prospectiva, la información de pacientes con diagnóstico de necrólisis epidérmica tóxica luego de aplicar una intervención con dosis y tratamiento bien definidos, con inmunoglobulina, ciclosporina A o metilprednisolona.

La distribución por edad fue variable, con presentación más frecuente en medianas entre los 40 y 50 años y límite superior hasta los 75 años. Hubo menor frecuencia del sexo masculino en cinco de los ocho estudios. El compromiso cutáneo fue mayor de $30 \%$ en siete estudios con medianas de extensión entre 40 y $60 \%$, lo que 


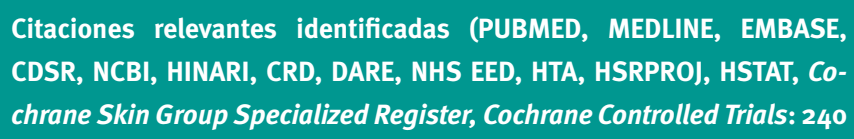

Estudios que llegaron a revisión detallada: 33

clínicos fase III incluidos: 8
Basado en título, resumen y palabras clave: citaciones excluidas: 207
Basado en la evaluación del texto completo: estudios excluidos: duplicados (22), no accesibilidad (1), no medida resultado mortalidad (1), cohorte retrospectiva (1)

FIGURA 1. Flujograma de estudios.

indica que la mayoría de los pacientes incluidos presentaron necrólisis epidérmica tóxica. El puntaje SCORTEN al ingreso se calculó en tres estudios en su medida absoluta y en uno de los estudios se determinó el porcentaje de muertes esperadas bajo esta escala. En otro estudio se tomó una medida de escala fisiológica de enfermedad aguda para determinar la gravedad de la enfermedad ${ }^{19}$.

Las dosis de medicamentos se ajustaron según el peso en seis estudios en que se utilizó ciclosporina A e inmunoglobulina $\mathrm{G}$ intravenosa. En dos estudios, uno con talidomida y otro con metilprednisolona, se utilizaron dosis fijas. Se administraron otros medicamentos tales como antibióticos, en quienes lo requirieron, cristaloides y agentes vasoactivos para el tratamiento de soporte de estas enfermedades. En un estudio, se incluyeron esteroides como tratamiento adicional a la ciclosporina o la ciclofosfamida. El tiempo de inicio del tratamiento fue en los primeros siete días, excepto en un estudio que fue a los 10 días del inicio de los síntomas ${ }^{21,22}$.

En cuatro de los estudios no se presentaron muertes a pesar del compromiso de superficie corporal de más del $50 \%$ 18,22,23,26. Los medicamentos utilizados en estos, fueron 160 a $240 \mathrm{mg}$ al día de metilprednisolona, 0,5 a $1 \mathrm{~g} / \mathrm{kg}$ diarios de inmunoglobulina intravenosa, $1,5 \mathrm{~g} /$ $\mathrm{kg}$ diarios de inmunoglobulina intravenosa y $3 \mathrm{mg} / \mathrm{kg}$ diarios de ciclosporina. También, se observó una mortalidad de menos de $10 \%$, comparada con la predicha de $35 \%$ en el grupo con inmunoglobulina a dosis de 400 $\mathrm{mg} / \mathrm{kg}$ diarios ${ }^{20}$. En contraste con esto, el uso de talidomida se asoció a una mortalidad del $83 \%$, con respecto al uso de placebo (30 \%), razón por la cual el estudio se detuvo al completar la mitad del tamaño muestral $\left(\mathrm{RR}=2,78 \mathrm{IC}_{95 \%} 1,04-7,4\right)^{19}$. Del mismo modo, el uso de altas dosis de inmunoglobulina ( $\mathrm{g} / \mathrm{kg}$ al día) se asoció a una mortalidad de $32 \%$, comparada con una predicha de $21 \%$ por escalas pronósticas (TABLA 1) ${ }^{17}$.

En un estudio con ciclosporina, no se produjeron muertes con una mediana de SCORTEN de 1 (rango intercuartílico, 0-2 \%) y una mortalidad esperada de $3,8 \%$ (rango intercuartílico, 1,1-12,19\%) ${ }^{26}$. En dos estudios con inmunoglobulina intravenosa y metilprednisolona, se hizo un cálculo de SCORTEN basado en dos parámetros presentes en los artículos (edad y porcentaje de superficie corporal comprometida), encontrándose una mortalidad de o $\%$ a pesar de una predicha de $6,9 \%$ (rango intercuartílico, 3,8-12,19 \%) y de 12,19\% (rango intercuartílico, 3,8-12,19\%), respectivamente. Aun así, estos últimos valores estarían subestimando el efecto del tratamiento.

Otros dos trabajos fueron experimentos clínicos con un número reducido de pacientes. En un estudio se comparó la ciclosporina A con un control histórico con ciclofosfamida ${ }^{22}$. Hubo mayor porcentaje de superficie corporal comprometida en el grupo con ciclospo- 


\begin{tabular}{|c|c|c|c|c|c|}
\hline Estudio & Medicamento & $\begin{array}{l}\text { Puntaje al } \\
\text { ingreso }^{¥}\end{array}$ & $\begin{array}{c}\text { Mortalidad } \\
\text { observada n } \\
\text { (\%) }\end{array}$ & $\begin{array}{c}\text { Mortalidad } \\
\text { esperada por } \\
\text { escala }^{¥}\end{array}$ & Eventos adversos \\
\hline \multirow{2}{*}{$\begin{array}{l}\text { Wolkenstein } \\
\text { et al., } 1998\end{array}$} & $\mathrm{~T}$ & $\begin{array}{c}11,5 \\
(6-19)^{\star \star}\end{array}$ & $10(83)$ & NA & Muerte en 10 pacientes \\
\hline & Placebo & $\begin{array}{c}10,5 \\
(6-17)^{\star \star}\end{array}$ & $3(30)$ & NA & ------ \\
\hline $\begin{array}{l}\text { Tripathi, et } \\
\text { al., } 2000\end{array}$ & M-PD & ND & o & $\begin{array}{c}12.9 \\
(3,8-12,9)^{\star}\end{array}$ & Ninguno \\
\hline \multirow{2}{*}{$\begin{array}{l}\text { Arévalo, et } \\
\text { al., } 2000\end{array}$} & CyA + CS & ND & o & NA & $\begin{array}{l}\text { Sepsis en } 8 \text { casos y falla } \\
\text { orgánica en } 2\end{array}$ \\
\hline & $\mathrm{CP}+\mathrm{CS}$ & ND & $3(50)$ & NA & $\begin{array}{l}\text { Leucopenia, } 3 \text { con falla } \\
\text { orgánica, } 5 \text { con sepsis }\end{array}$ \\
\hline $\begin{array}{l}\text { Campione, } \\
\text { et al., } 2003\end{array}$ & IgG IV & $2,5(2-4)$ & $1(10)$ & $\begin{array}{c}20,4 \\
(12,2-62,2)\end{array}$ & $\begin{array}{c}\text { Cefalea, náusea y alteraciones } \\
\text { gastrointestinales }\end{array}$ \\
\hline $\begin{array}{l}\text { Bachot, et } \\
\text { al., } 2003\end{array}$ & IgG IV & $2(1-3)$ & $11(32,3)$ & $\begin{array}{c}12,1 \\
(3,8-32,3)\end{array}$ & Deterioro función renal \\
\hline $\begin{array}{l}\text { Al-Mutairi, } \\
\text { et al., } 2004\end{array}$ & IgG IV & ND & o & $\begin{array}{c}6,9 \\
(3,8-12,19)^{\star}\end{array}$ & Ninguno \\
\hline \multirow{3}{*}{$\begin{array}{l}\text { Paquet, et } \\
\text { al, } 2005\end{array}$} & IgG IV & ND & $1(50)$ & NA & $\begin{array}{l}1 \text { caso de insuficiencia renal } \\
\text { complicado con SAMR }\end{array}$ \\
\hline & СуA & ND & $1(50)$ & NA & $\begin{array}{l}\text { Muerte por sepsis por } \\
\text { pseudomona. }\end{array}$ \\
\hline & Soporte & ND & 0 & NA & ------ \\
\hline $\begin{array}{l}\text { Valerye, et } \\
\text { al., } 2010\end{array}$ & СуA & $1(0-2)$ & 0 & $\begin{array}{c}3,8 \\
(1,1-12,19)\end{array}$ & $\begin{array}{l}\text { Alucinaciones, Síndrome } \\
\text { de encefalopatía posterior } \\
\text { reversible, neutropenia } \\
\text { transitoria, neumopatía } \\
\text { hospitalaria. Otros, } \\
\text { hipertensión arterial, } \\
\text { disminución leve de función } \\
\text { renal, neuropatía sensitiva. }\end{array}$ \\
\hline
\end{tabular}

$\star \star$ Escala fisiológica aguda simplificada -SAPS- (Promedio y rango);

${ }^{\ddagger}$ Mediana y rango intercuartílico; ${ }^{\star}$ Medida calculada bajo dos parámetros (edad y porcentaje superficie corporal comprometida); T: talidomida; CyA: ciclosporina A; CP: ciclofosfamida; IgG IV: inmunoglobulina G intravenosa; CS: corticosteroides; ND: no disponible; NA: no aplica.

TABLA 1. Medicamento antiinflamatorio utilizado, puntaje al ingreso, mortalidad observada y esperada, y eventos adversos relacionados con tratamiento por estudio. 
rina con una mediana de $90 \%$ (rango intercuartílico, 85-90\%), en comparación con ciclofosfamida (77,5\%; rango intercuartílico, 60-90\%). No obstante, la mortalidad en el primer grupo fue de o \%, comparada con una de $50 \%$ en el control histórico. En otro estudio se comparó el uso de ciclosporina en dos pacientes, inmunoglobulina en otros dos y se compararon con un control histórico de dos pacientes ${ }^{21}$. La superficie corporal comprometida fue de 50 y $60 \%$ (inmunoglobulina y ciclosporina, respectivamente) y se observó una mortalidad de $50 \%$ (un paciente) para cada fármaco, mientras que la mortalidad fue de o \% en el control histórico. La mortalidad observada, la mortalidad esperada y el reporte de eventos adversos, se encuentran en la TABLA 1.

\section{Discusión}

No existe un tratamiento específico para el síndrome de Stevens-Johnson o la necrólisis epidérmica tóxica. En la actualidad, las únicas recomendaciones claras son suspender el fármaco desencadenante y usar medidas de soporte que buscan controlar las complicaciones asociadas con estas entidades ${ }^{1,5,10}$. La mortalidad en este grupo de enfermedades está determinada por la gravedad del compromiso cutáneo al momento de la presentación, la edad, la frecuencia cardiaca, la presencia de neoplasia maligna y los niveles de nitrógeno ureico, bicarbonato y glucemia ${ }^{13}$. Considerando lo anterior, se han publicado cada vez más reportes donde se sugiere el beneficio de ciertos medicamentos antiinflamatorios.

Previamente se hizo una revisión sistemática en la cual se incluyeron ensayos clínicos de fase III, en la que solo se encontró un ensayo clínico de asignación aleatoria y controlado con placebo, al igual que la publicación de Mahumdar, et al. ${ }^{15}$, por lo cual se decidió incluir estudios experimentales sin grupo control con el objetivo de recopilar la mejor información disponible hasta la fecha y teniendo en cuenta que no hay ningún medicamento aprobado. Un ensayo clínico se define como un estudio prospectivo que compara el efecto y valor de una intervención o experimento, contra un $\operatorname{control}^{27}$.

En el caso de los estudios sin grupo control, se comparó su resultado con la escala pronóstica establecida por Batsuji-Garin, et al. ${ }^{13}$. Esta escala fue elaborada bajo un estudio observacional de 165 pacientes con diagnóstico de necrólisis epidérmica tóxica o síndrome de Stevens-Johnson, y determinó los factores de predicción independientes para mortalidad. Posteriormente, se validó la escala a los cinco días de hospitalización, observándose una mortalidad de o $\%\left(\mathrm{IC}_{95 \%} \mathrm{O}-8,2 \%\right)$ cuando el SCORTEN fue 0-1; 6,7\% (IC ${ }_{95 \%} 1,4-18,3$ ), con puntaje de 2; 23,8 \% ( IC $_{95 \%} 8,2-47,2 \%$ ), cuando el puntaje fue 3; 35,3 \% (IC ${ }_{95 \%}$ 14,2-61,7), cuando alcanzó 4, y 77,8 \%
(IC $\left._{95 \%} 52,4-93,6\right)$, cuando fue de 50 más. Se estableció la probabilidad de muerte como $\mathrm{P}($ death $)=\mathrm{e}^{\text {logit }} / 1+\mathrm{e}^{\text {logit }}$, donde logit fue $-4,448+1,237$ (SCORTEN) con un porcentaje de concordancia excelente entre la mortalidad observada y la esperada ${ }^{13}$.

En este mismo estudio, se establecieron factores pronósticos independientes para mortalidad. Para el caso de la edad, tener 40 años o más aumentó la probabilidad de morir en 1,7 veces (OR=2,7; $\left.\mathrm{IC}_{95 \%} 1,0-7,5 ; \mathrm{p}=0,05\right)$, y para el porcentaje de superficie corporal comprometida, la mortalidad se aumentó más de dos veces cuando fue igual o mayor a $10 \%$ (OR de 2,9; $\mathrm{IC}_{95 \%} 0,9$ a 8,8, para un porcentaje entre 10 y $30 \%$, y OR de 3,3; IC ${ }_{95 \%}$ de 1,29,6, en el caso de un porcentaje mayor de $30 \%$; $p=0,04$ ), lo que mostró que, con solo dos puntos de la escala, la probabilidad de morir era 4,1 veces respecto a los que no tenían ninguno $\left(\mathrm{OR}=4,1 ; \mathrm{IC}_{95 \%} \mathrm{O}, 5-35,2\right)$. En nuestra revisión, estas dos variables se observaron a lo largo de todos los estudios, lo cual permitió tener una medida gruesa de las mortalidades esperadas en estos grupos, pudiéndolas comparar con las mortalidades observadas en los estudios.

La principal limitación de los estudios incluidos fue el tamaño tan pequeño de la muestra, pero hay que tener en cuenta la baja incidencia de la enfermedad, lo que hace difícil alcanzar un tamaño de muestra que permita encontrar diferencias entre la intervención y el placebo; además, los autores de los diferentes estudios no consideraron ética la comparación con placebo. La calidad de los artículos dada por la asignación aleatoria y enmascaramiento, solo fue evaluada en un estudio puesto que fue el único ensayo clínico de asignación aleatoria controlado con placebo ${ }^{19}$. Dicho trabajo se detuvo a la mitad del tamaño de muestra alcanzado por evidenciarse aumento de la mortalidad en uno de los grupos. En los otros estudios no se pudo evaluar la calidad debido a que fueron experimentales sin grupo control, y se valieron de escalas de predicción para establecer el posible beneficio de la intervención. Tampoco hubo enmascaramiento de la intervención para los investigadores que evaluaron los resultados; sin embargo, incluyeron un resultado muy fuerte como lo es la mortalidad. Todo lo anterior desestimó la posibilidad de hacer un metaanálisis. La mayoría de los estudios fueron ensayos abiertos sin grupo control, que podrían clasificarse como estudios observacionales. Sin embargo, se establecieron criterios de inclusión, se aplicó una intervención y se hizo seguimiento en el tiempo, lo cual aplica para un estudio experimental. En ausencia de un grupo control, se tomó como medida de comparación la superficie corporal comprometida como factor pronostico ${ }^{1}$. La escala SCORTEN representa uno de los factores que predicen mortalidad ${ }^{13}$. 
Asimismo, los diferentes medicamentos utilizados y las diferentes dosis a lo largo de los estudios limitan las recomendaciones respecto al tratamiento actual, pero se pudo establecer que en los trabajos con metilprednisolona, e inmunoglobulina y ciclosporina a ciertas dosis, no hubo mortalidad, a pesar de la gran extensión de superficie corporal comprometida. Es importante resaltar también que en la mayoría de los estudios se inició el tratamiento antiinflamatorio entre los dos y cinco días después de iniciados los síntomas, lo que podría influir en el resultado debido a que no se reportan los pacientes que murieron en las primeras 24 a 48 horas, los cuales pudieron tener un puntaje de riesgo más alto.

Con todas las consideraciones anteriores y teniendo en cuenta que la SCORTEN es una escala de pronóstico que predice mortalidad, parece existir cierto beneficio de los tratamientos con metilprednisolona, inmunoglobulina y ciclosporina. Se abre la posibilidad de hacer ensayos clínicos controlados comparando las diferentes alternativas, haciendo énfasis en que, a pesar de que no existe un tratamiento aprobado para estas enfermedades, no pareciera ético tomar la opción de no ofrecer algún tratamiento antiinflamatorio o inmunomodulador.

\section{Referencias}

1. Harr T, French L. Toxic epidermal necrolysis and Stevens-Johnson syndrome. Orphanet J Rare Dis. 2010;5:39.

2. Crosi A, Borges GS, Estévez CF. Reacciones adversas medicamentosas graves: síndrome de Stevens-Johnson y necrólisis epidérmica tóxica. Rev Med Uruguay. 2004;20:172-7.

3. Gerull R, Nelle M, Schaible T. Toxic epidermal necrolysis and Stevens-Johnson syndrome: A review. Crit Care Med. 2011;39:1521-32

4. Mesa T. Síndrome de Stevens Johnson posterior al uso de carbamazepina en paciente esquizofrénico. Reporte de caso. Psiquiatriacom. 2011;15: 1-16.

5. Chen P, Lin J-J, Lu C-S, Ong C-T, Hsieh PF, Yang C-C, et al. Carbamazepine-induced toxic effects and HLA-B ${ }^{\star} 1502$ screening in Taiwan. N Engl J Med. 2011;364:1126-33.

6. Fournier S, Bastuji-Garin S, Mentec H, Revuz J, Roujeau J. Toxic epidermal necrolysis associated with Mycoplasma pneumoniae infection. Eur J Clin Microbiol Infect Dis . 1995;14:558-9.

7. Mulvey J, Padowitz A, Lindley-Jones M, Nickels R. Mycoplasma pneumoniae associated with Stevens Johnson syndrome. Anaesth Intensive Care. 2007;35:414-7.

8. Schalock PC, Dinulos JGH. Mycoplasma pneumoniae-induced Stevens-Johnson syndrome without skin lesions: Fact or fiction? J Am Acad Dermatol. 2005;52:312-5.

9. Auquier-Dunant A, Mockenhaupt M, Naldi L, Correia O, Schroder $\mathrm{W}$, Roujeau J-C, et al. Correlations between clinical patterns and causes of erythema multiforme majus, Stevens-Johnson syndrome, and toxic epidermal necrolysis: Results of an international prospective study. Arch Dermatol. 2002;138:1019-24.

10. Tartarone A, Lerose R. Stevens-Johnson syndrome and toxic epidermal necrolysis: What do we know? Ther Drug Monit. 2010;32:669-72.
11. Roujeau J-C, Kelly JP, Naldi L, Rzany B, Stern RS, Anderson T, et al. Medication use and the risk of Stevens-Johnson syndrome or toxic epidermal necrolysis. N Engl J Med. 1995;333:1600-8.

12. La Grenade L, Lee L, Weaver J, Bonnel R, Karwoski C, Governale $\mathrm{L}$, et al. Comparison of reporting of Stevens-Johnson syndrome and toxic epidermal necrolysis in association with selective COX-2 inhibitors. Drug Safety. 2005;28:917-24.

13. Bastuji-Garin S, Fouchard N, Bertocchi M, Roujeau J-C, Revuz J, Wolkenstein P. SCORTEN: A severity-of-illness score for toxic epidermal necrolysis. J Invest Dermatol. 2000;115 (2):149-53.14.

14. Guegan S, Bastuji-Garin S, Poszepczynska-Guigne E, Roujeau J-C, Revuz J. Performance of the SCORTEN during the first five days of hospitalization to predict the prognosis of epidermal necrolysis. J Invest Dermatol. 2005;126:272-6.

15. Majumdar S, Mockenhaupt M, Roujeau J, Townshend A. Interventions for toxic epidermal necrolysis. Cochrane Database Syst Rev. 2002;4:CD991435.

16. Shortt R, Gómez M, Mittman N, Cartotto R. Intravenous immunoglobulin does not improve outcome in toxic epidermal necrolysis. J Burn Care Rehabil. 2004;25:246-55.

17. Bachot N, Revuz J, Roujeau J-C. Intravenous immunoglobulin treatment for Stevens-Johnson syndrome and toxic epidermal necrolysis: A prospective noncomparative study showing no benefit on mortality or progression. Arch Dermatol. 2003;139:33-6.

18. Tripathi A, Ditto AM, Grammer LC, Greenberger PA, McGrath KG, Zeiss CR, et al. Corticosteroid therapy in an additional 13 cases of Stevens-Johnson syndrome: A total series of 67 cases. Allergy Asthma Proc. 2000;21:101-5.

19. Wolkenstein P, Latarjet J, Roujeau J-C, Duguet C, Boudeau S, Vaillant $\mathrm{L}$, et al. Randomized comparison of thalidomide versus placebo in toxic epidermal necrolysis. Lancet. 1998;352:1586-9.

20. Campione E, Marulli GC, Carrozzo AM, Chimenti MS, Costanzo A, Bianchi L. High-dose intravenous immunoglobulin for severe drug reactions: efficacy in toxic epidermal necrolysis. Acta Derm Venereol. 2003;83:430-2.

21. Paquet P, Jacob E, Damas P, Pirson J, Piérard G. Analytical quantification of the inflammatory cell infiltrate and CD95R expression during treatment of drug-induced toxic epidermal necrolysis. Arch Dermatol Res. 2005;297:266-73.

22. Arévalo J, Llorente JA, González-Herrada C, Jiménez-Reyes J. Treatment of toxic epidermal necrolysis with cyclosporin A. J Trauma. 2000;48:473-8.

23. Al-Mutairi N, Arun J, Osama N-E, Amr Z, Mazen A-S, Ibtesam E-A, et al. Prospective, noncomparative open study from Kuwait of the role of intravenous immunoglobulin in the treatment of toxic epidermal necrolysis. Int J Dermatol. 2004;43:847-51.

24. Araki Y, Sotozono C, Inatomi T, Ueta M, Yokoi N, Ueda E, et al. Successful treatment of Stevens-Johnson syndrome with steroid pulse therapy at disease onset. Am J Ophthalmol . 2009;147:1004-11.

25. Eisen E, Fish J, Shear N. Management of drug-induced toxic epidermal necrolysis. Cutan Med Surg. 2000;4:96-102.

26. Valeyrie-Allanore L, Wolkenstein P, Brochard L, Ortonne N, Maitre B, Revuz J, et al. Open trial of ciclosporin treatment for StevensJohnson syndrome and toxic epidermal necrolysis. Br J Dermatol. 2010;163:487-53.

27. Friedman LM, Furberg CD, DeMets DL: Basic study design. In: Fundamentals of Clinical Trials, 4th Ed., edited by Friedman LM, Furberg CD, DeMets DL, New York, Springer, 2010, pp 68-71 